\title{
Extralaryngeal branching of the recurrent laryngeal nerve: a meta-analysis of 28,387 nerves
}

\author{
Brandon Michael Henry ${ }^{1,2}$. Jens Vikse ${ }^{1,2}$ - Matthew J. Graves ${ }^{1,2} \cdot$ Silvia Sanna ${ }^{3}$. \\ Beatrice Sanna $^{4}$ - Iwona M. Tomaszewska ${ }^{5}$ - R. Shane Tubbs ${ }^{6}$. \\ Krzysztof A. Tomaszewski ${ }^{1,2}$
}

Received: 25 February 2016 / Accepted: 24 May 2016 / Published online: 2 June 2016

(C) The Author(s) 2016. This article is published with open access at Springerlink.com

\begin{abstract}
Introduction The recurrent laryngeal nerves (RLN) are branches of the vagus nerve that go on to innervate most of the intrinsic muscles of the larynx. Historically, the RLN has been considered to branch after it enters the larynx, but numerous studies have demonstrated that it often branches before. The wide variability of this extralaryngeal branching (ELB) has significant implications for the risk of iatrogenic injury. We aimed to assess the anatomical characteristics of ELB comprehensively.

Methods Articles on the ELB of the RLN were identified by a comprehensive database search. Relevant data were extracted and pooled into a meta-analysis of the prevalence of branching, branching pattern, distance of ELB point from the larynx, and presence of positive motor signals in anterior and posterior ELB branches.
\end{abstract}

Electronic supplementary material The online version of this article (doi:10.1007/s00423-016-1455-7) contains supplementary material, which is available to authorized users.

Brandon Michael Henry

bmhenry55@gmail.com

1 International Evidence-Based Anatomy Working Group, 12 Kopernika St, 31-034 Krakow, Poland

2 Department of Anatomy, Jagiellonian University Medical College, 12 Kopernika St, 31-034 Krakow, Poland

3 Department of Surgical Sciences, University of Cagliari, S.S. 554, Bivio Sestu, 09042 Monserrato, CA, Sardinia, Italy

4 Faculty of Medicine and Surgery, University of Cagliari, S.S. 554, Bivio Sestu, 09042 Monserrato, CA, Sardinia, Italy

5 Department of Medical Education, Jagiellonian University Medical College, 16 św. Łazarza Street, 31-530 Krakow, Poland

6 Seattle Science Foundation, 550 17th Ave, James Tower, Suite 600, Seattle, WA 28122, USA
Results A total of 69 articles ( $n=28,387$ nerves) from both intraoperative and cadaveric modalities were included in the meta-analysis. The overall pooled prevalence of ELB was $60.0 \%$ (95\% CI 52.0-67.7). Cadaveric and intraoperative subgroups differed with prevalence rates of $73.3 \%(95 \% \mathrm{CI}$ 61.0-84.0) and $39.2 \%$ (95\% CI 29.0-49.9), respectively. Cadavers most often presented with a ELB pattern of bifurcation, with a prevalence of $61.1 \%$, followed by no branching at $23.4 \%$. Branching of the RLN occurred most often at a distance of $1-2 \mathrm{~cm}$ ( $74.8 \%$ of cases) prior to entering the larynx. A positive motor signal was most often noted in anterior RLN branches $(99.9 \%$ ) but only in $1.5 \%$ of posterior branches. Conclusions The anatomy of the RLN is highly variable, and ELB is likely to have been underreported in intraoperative studies. Because of its high likelihood, the possibility of ELB needs to be assessed in patients to prevent iatrogenic injury and long-term postoperative complications.

Keywords Recurrent laryngeal nerve $\cdot$ Extralaryngeal branching $\cdot$ Thyroid $\cdot$ Surgery $\cdot$ Anatomic variations

\section{Introduction}

The recurrent laryngeal nerves (RLN) are branches of the vagus nerve, which classically arise in the inferior neck and innervate the intrinsic muscles of the larynx except for the cricothyroid muscle [1]. However, it has recently been shown that the RLN can also contribute significantly to the innervation of the cricothyroid muscle [2]. Its terminal branches must split in order to innervate their respective muscles, but this branching can occur anywhere from several centimeters from the inferior rim of the cricothyroid joint to within the larynx itself [3-8]. The terminal branch of the RLN as it courses superior to the cricothyroid joint is commonly deemed the 
inferior laryngeal nerve. It is of paramount importance that the RLN and its extralaryngeal branches (ELB), if present, are carefully dissected and identified during procedures in the anterior neck. Failure to identify these neural structures, or inadequate knowledge of their variability, can lead to an increased incidence of iatrogenic nerve injury [9]. As described by Kandil et al. in 2011 [10], the RLN typically branches superior to the inferior thyroid artery and posterolaterally to the ligament of Berry, and this is the location where the nerve is most susceptible to injury. If the posterior branch is identified and believed to be the sole RLN, the anterior branch is particularly vulnerable to injury when the capsular dissection approach to thyroidectomy is used [4]. If the anterior branch is identified first, it is more likely that the surgeon will find the posterior branch during capsular dissection, thereby preventing injury [4]. The likelihood of lesion to the anterior branch is particularly important because there is a high risk of vocal cord palsy and long-term complications from its injury.

Data on the prevalence of ELB have been debated for years, with reported prevalence rates ranging widely from around $5 \%$ [11] to $100 \%$ [12-21]. The rates also differ depending on whether the studies were conducted intraoperatively or on cadavers. Since the RLN is very susceptible to injury in a multitude of procedures, a thorough and complete understanding of its variability and the associated implications is crucial for preventing iatrogenic injuries and long-term complications. The aim of our analysis was to provide a comprehensive and evidence-based assessment of the ELB of the RLN. An accurate and complete assessment of the ELB is necessary to provide a complete understanding of the risk factors associated with neck surgery and the vital importance of taking precautionary measures to prevent injury-related complications.

\section{Methods}

\section{Search strategy}

To identify articles for inclusion in the meta-analysis, searches were performed through December 2015 in the following databases: PubMed, EMBASE, ScienceDirect, China National Knowledge Infrastructure (CNKI), SciELO, BIOSIS, and Web of Science. The comprehensive search strategy applied to PubMed is presented in Table 1. No date or language restrictions were imposed. In order to identify additional studies eligible for the meta-analysis, the references of all included articles were thoroughly searched. Throughout the meta-analysis, the Preferred Reporting Items for Systematic Reviews and Meta-Analyses (PRISMA) guidelines were strictly followed (Online Resource 1) [22]. Our study was prospectively registered in the PROSPERO database (CRD42015026096).

\section{Eligibility assessment}

Eligibility of studies for inclusion in the meta-analysis was assessed by three independent reviewers (JV, MJG, and SS). All cadaveric or intraoperative studies that reported extractable prevalence data with respect to rate of ELB were included. The exclusion criteria included case reports, case series, letters to the editor, or conference abstracts. Studies on human fetuses or involving patients with congenital anomalies of or trauma to the head and neck region were also excluded. All studies published in languages not fluently spoken by any of the authors were translated by medical professionals fluent in both English and the language of the manuscript. Any disagreements between reviewers arising during the eligibility assessment process were resolved by consensus.

\section{Data extraction}

Data from the included studies were independently extracted by three reviewers (BMH, JV, and SS). The extracted data included year, country, sample size (number of nerves), prevalence of ELB, symmetry of ELB, type of ELB (no branching, bifurcation, trifurcation, multiple branches), the distance from the ELB site to the inferior rim of the cricothyroid joint (0-1, $1-2,2-3,3-4 \mathrm{~cm}$ ), and the intraoperative electrophysiologically assessed prevalence of positive motor signals in the anterior and posterior branches of ELB RLNs. In the event of any discrepancies in the data, the authors of the original were contacted for clarification when possible.

\section{Statistical analysis}

The single-categorical and multi-categorical pooled prevalence rates of the ELB of the RLN were calculated by BMH and JV using MetaXL version 2.0 by EpiGear Pty Ltd. (Wilston, Queensland, Australia) [23]. A random effects model was used for all statistical analyses. Heterogeneity was assessed by both the chi ${ }^{2}$ test and the $I^{2}$ statistic. For the chi ${ }^{2}$ test, a $p$ value of $<0.10$ for Cochran's $Q$ served as an indicator of significant heterogeneity among the studies analyzed [24]. The results of the $I^{2}$ statistic were interpreted as follows: 0-40\% might not be important; 30-60\% could indicate moderate heterogeneity; 50-90\% could indicate substantial heterogeneity; and 75$100 \%$ could represent considerable heterogeneity [24].

Subgroup analysis was performed on the basis of type of study (cadaveric vs. intraoperative), study design (prospective vs. retrospective), geographical origin of the study, gender, and side (left vs. right). Data on subgroups was maximally extracted based on its availability within the analyzed studies. Significant differences between analyzed groups were determined by their confidence intervals. If the confidence intervals of any two rates overlapped, the differences were regarded as statistically insignificant [23]. Lastly, sensitivity was assessed 
Table 1 Search terms and strategy for PubMeb

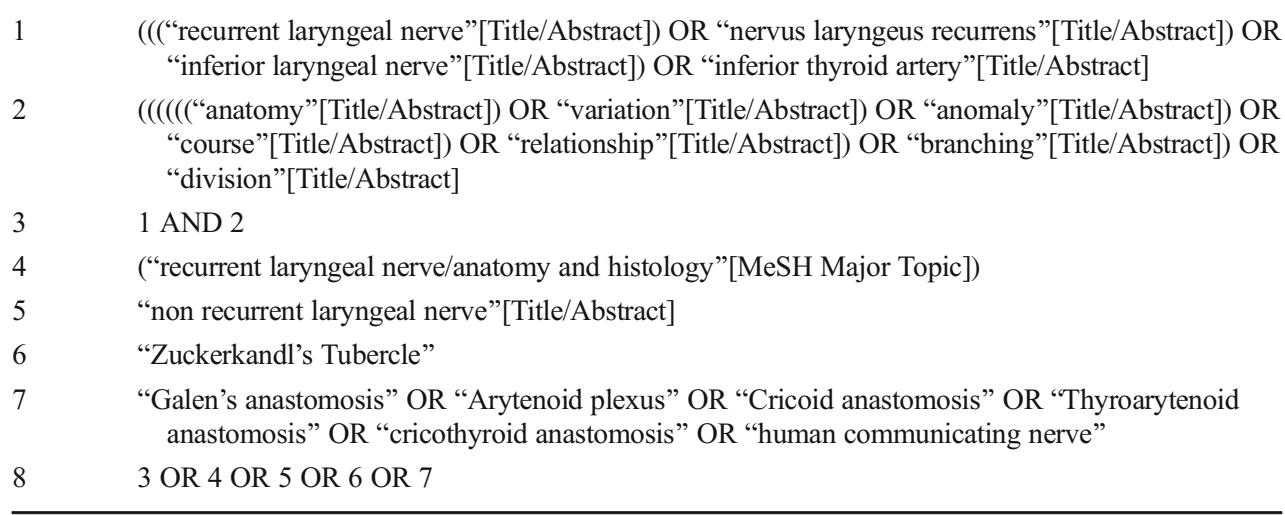

by a leave-one-out analysis to probe further for potential sources of heterogeneity.

\section{Results}

\section{Study identification}

The flow of studies through the meta-analysis is presented in Fig. 1. The search of the major electronic databases identified an initial 2795 articles, with a

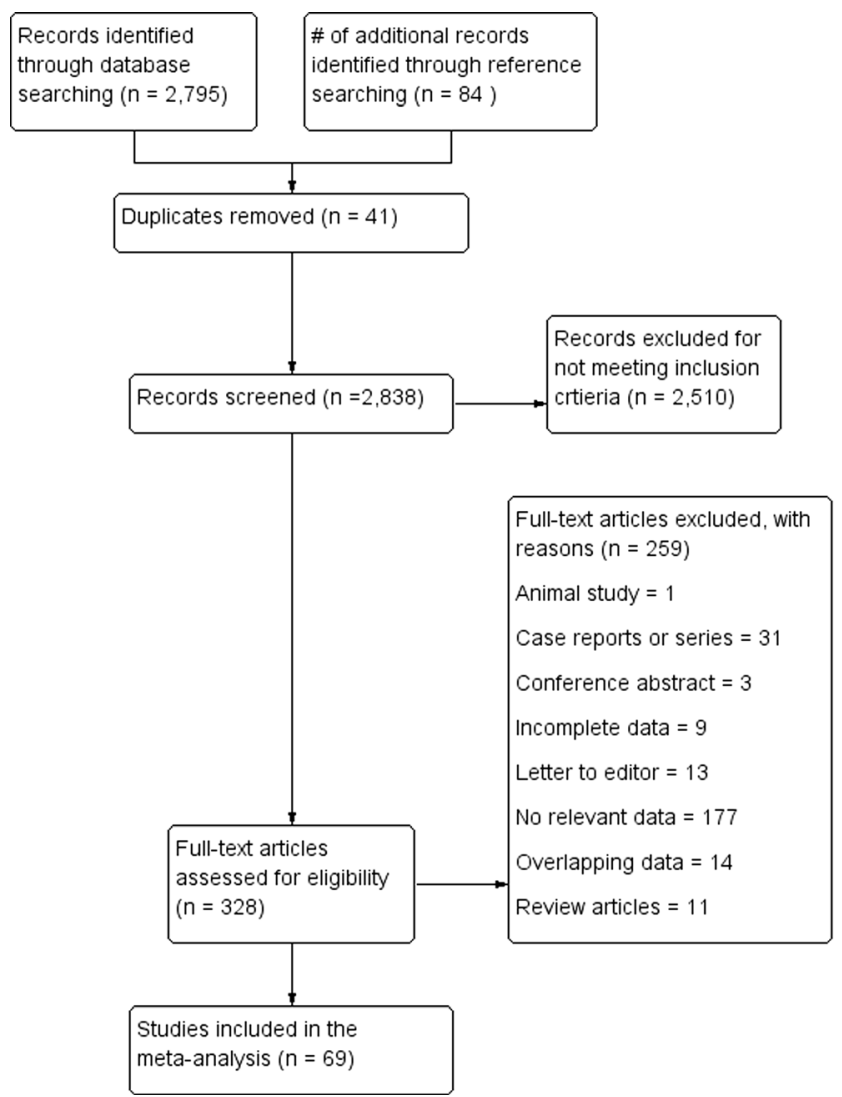

Fig. 1 PRISMA flow chart of study identification and inclusion in the meta-analysis further 84 identified in the search through the references of those studies. A total of 328 articles were assessed for eligibility using full texts, of which 259 were excluded and 69 were included in the meta-analysis.

\section{Characteristics of included studies}

The characteristics of the studies included in the meta-analysis are summarized in Table 2. A total of 69 studies [3-7, 9-21, 25-75] were included ( $n=28,387$ total nerves): 26 intraoperative, 42 cadaveric, and 1 that included both intraoperative and cadaveric subjects [50]. Among the intraoperative studies, 16 were prospective and 10 were retrospective. The dates of the included studies ranged from 1921 [13] to the end of year 2015 [30, 41, 66]. The studies demonstrated a wide range of geographical origin, with the most substantial contributions coming from Asia (22 studies), Europe (25), and North America (13). Fourteen studies in Chinese, one in French, one in Italian, and one in Portuguese were translated into English and included in our analysis.

\section{Prevalence of extralaryngeal branching}

A total of 69 studies ( $n=28,387$ nerves) reported data on ELB prevalence. The overall pooled prevalence rate of ELB was $60.0 \%$ (95 \% CI 52.0-67.7) (Fig. 2). Subgroup analysis by type of study revealed significant differences in the prevalence of ELB between cadaveric (73.3\%; $95 \%$ CI 61.0-84.0) and intraoperative (39.2\%; $95 \%$ CI 29.0-49.9) studies. No significant differences were found between subgroups with respect to side (left vs. right), gender, or geographical origin. Details of subgroup analyses are presented in Table 3. No significant differences were observed in the sensitivity analysis.

A total of six studies ( $n=641$ subjects) reported data on the symmetry of the RLN with respect to ELB. The RLN was found to be symmetrical in $36.5 \%$ (95\% CI 16.1-59.6) of individuals $\left(I^{2}=96.6 \%\right.$ (95\% CI 94.5-97.9); $\left.p<0.001\right)$. 
Table 2 Table of included studies

\begin{tabular}{|c|c|c|c|c|}
\hline Study & Country & Type & $\begin{array}{l}n \text { (no. of } \\
\text { nerves) }\end{array}$ & $\begin{array}{l}\% \text { of } \\
\text { ELB }\end{array}$ \\
\hline Al-Salihi and Dabbagh [25] & Iraq & $\mathrm{C}$ & 212 & 25.5 \\
\hline Altorjay et al. [26] & Hungary & IP & 1023 & 51.5 \\
\hline Ardito et al. [27] & Italy & IP & 2615 & 72.4 \\
\hline Armstrong and Hinton [28] & USA & $\mathrm{C}$ & 100 & 73.0 \\
\hline Asgharpour et al. [3] & Spain & $\mathrm{C}$ & 284 & 54.6 \\
\hline Barczyński et al. [29] & Poland & IP & 302 & 22.2 \\
\hline Barczyński et al. [30] & Poland & IP & 2500 & 24.5 \\
\hline Bargy et al. [31] & France & $\mathrm{C}$ & 56 & 10.7 \\
\hline Beneragama and Serpell [4] & Australia & IP & 213 & 40.4 \\
\hline Bowden [32] & Great Britain & $\mathrm{C}$ & 54 & 77.8 \\
\hline Cakir et al. [33] & Turkey & $\mathrm{C}$ & 130 & 58.5 \\
\hline Cernea et al. [34] & Brazil & IR & 2154 & 64.5 \\
\hline Chang [12] & China & $\mathrm{C}$ & 50 & 100 \\
\hline Chen et al. [35] & China & $\mathrm{C}$ & 90 & 68.9 \\
\hline Chen et al. [36] & China & $\mathrm{C}$ & 94 & 69.1 \\
\hline Clader et al. [37] & USA & $\mathrm{C}$ & 50 & 58.0 \\
\hline Dai et al. [38] & China & IR & 339 & 59.9 \\
\hline Dilworth [13] & England & $\mathrm{C}$ & 66 & 100 \\
\hline Fontenot et al. [39] & USA & IR & 719 & 36.7 \\
\hline Gurleyik [40] & Turkey & IP & 200 & 27.0 \\
\hline Gurleyik [41] & Turkey & IP & 185 & 33.0 \\
\hline Hisham and Lukman [42] & Malaysia & IP & 490 & 34.1 \\
\hline Hsu et al. [14] & China & $\mathrm{C}$ & 177 & 100 \\
\hline Iqbal and Zumair [43] & Pakistan & IR & 93 & 58.1 \\
\hline Jiang et al. [44] & China & IR & 292 & 63.4 \\
\hline Kandil et al. [10] & USA & IP & 310 & 42.9 \\
\hline Katz and Nemiroff [45] & USA & IP & 1177 & 63.5 \\
\hline Keros and Nemanić [15] & Croatia & $\mathrm{C}$ & 300 & 100 \\
\hline King and Gregg [46] & USA & $\mathrm{C}$ & 43 & 27.9 \\
\hline Kulekci et al. [47] & Turkey & $\mathrm{C}$ & 194 & 80.4 \\
\hline Kuo et al. [48] & China & $\mathrm{C}$ & 100 & 62.0 \\
\hline Laux and Guerrier [49] & France & $\mathrm{C}$ & 200 & 43.0 \\
\hline Lu et al. [50] & China & $\mathrm{C}+\mathrm{IR}$ & 66 & 27.3 \\
\hline Makay et al. [9] & Turkey & IP & 253 & 24.1 \\
\hline Matubis et al. [51] & Philippines & $\mathrm{C}$ & 108 & 14.8 \\
\hline Moreau et al. [52] & France & $\mathrm{C}$ & 34 & 29.4 \\
\hline Morrison [53] & USA & $\mathrm{C}$ & 200 & 43.0 \\
\hline Nemiroff and Katz [5] & USA & IP & 153 & 41.2 \\
\hline Ngo Nyeki et al. [54] & $\begin{array}{l}\text { Cameroon and } \\
\text { Gabon }\end{array}$ & IP & 62 & 9.7 \\
\hline Nguyen et al. [55] & France & $\mathrm{C}$ & 60 & 86.7 \\
\hline Norland [56] & USA & $\mathrm{C}$ & 62 & 96.8 \\
\hline Page et al. [57] & France & IP & 403 & 19.4 \\
\hline Pascoal et al. [58] & Brazil & $\mathrm{C}$ & 44 & 70.5 \\
\hline Pichler and Gisel [16] & Austria & $\mathrm{C}$ & 100 & 100 \\
\hline Pradeep et al. [59] & India & IR & 583 & 30.5 \\
\hline Prior and Fasce [60] & Italy & $\mathrm{C}$ & 100 & 11.0 \\
\hline Reed [11] & USA & $\mathrm{C}$ & 506 & 5.3 \\
\hline
\end{tabular}

Table 2 (continued)

\begin{tabular}{|c|c|c|c|c|}
\hline Study & Country & Type & $\begin{array}{l}n \text { (no. of } \\
\text { nerves) }\end{array}$ & $\begin{array}{l}\% \text { of } \\
\text { ELB }\end{array}$ \\
\hline de Souza 1981 [61] & Brazil & $\mathrm{C}$ & 98 & 25.5 \\
\hline Rueger [17] & USA & $\mathrm{C}$ & 19 & 100 \\
\hline Rustad [62] & USA & $\mathrm{C}$ & 200 & 43.0 \\
\hline Salama and McGrath [6] & Australia & $\mathrm{C}$ & 144 & 65.3 \\
\hline Schweizer and Dörfl [7] & Switzerland & $\mathrm{C}$ & 42 & 88.1 \\
\hline Serpell et al. [63] & Australia & IP & 838 & 25.7 \\
\hline Serpell [64] & Australia & IR & 977 & 24.7 \\
\hline Shao et al. [65] & China & IP & 4241 & 8.6 \\
\hline Shao et al. [66] & China & IR & 2869 & 11.2 \\
\hline She et al. [67] & China & $\mathrm{C}$ & 200 & 42.0 \\
\hline She et al. [18] & China & $\mathrm{C}$ & 100 & 100 \\
\hline Sun et al. [68] & China & $\mathrm{C}$ & 100 & 94.0 \\
\hline Sunderland and Swaney [69] & Australia & $\mathrm{C}$ & 130 & 70.0 \\
\hline Tang et al. [70] & China & $\mathrm{C}$ & 160 & 91.9 \\
\hline Wang et al. [71] & China & IR & 63 & 76.2 \\
\hline Weeks and Hinton [72] & USA & IR & 17 & 88.2 \\
\hline Williams [19] & England & $\mathrm{C}$ & 100 & 100 \\
\hline Yalcin et al. [73] & Turkey & $\mathrm{C}$ & 96 & 92.7 \\
\hline Yalcin et al. [74] & Turkey & $\mathrm{C}$ & 120 & 93.3 \\
\hline Yang et al. [20] & China & $\mathrm{C}$ & 90 & 100 \\
\hline Yuan [75] & China & $\mathrm{C}$ & 117 & 67.5 \\
\hline Zhou et al. [21] & China & $\mathrm{C}$ & 120 & 100 \\
\hline
\end{tabular}

$E L B$ extralaryngeal branching, $C$ cadaveric, $I P$ intraoperative prospective, $I R$ intraoperative retrospective

\section{Prevalence of the types of extralaryngeal branching}

A total of 47 studies ( $n=16,618$ nerves) reported data on the type of ELB of the RLN. Bifurcation was the most common pattern observed, with a pooled prevalence of $51.1 \%(95 \%$ CI 35.7-55.3) of nerves, followed by no branching, $42.0 \%$ (95\% CI 28.1-47.1) (Online Resource 2). Trifurcation and multiple branches were less common, with pooled prevalence rates of $4.7 \%$ (95\% CI 1.0-9.2) and $2.2 \%$ (95\% CI 0-5.9), respectively. As with the pooled prevalence of ELB, cadaveric and intraoperative studies differed significantly, the rate of bifurcation being significantly greater in cadaveric $(61.1 \%$; $95 \%$ CI 33.8-78.4) (Fig. 3) than intraoperative (37.6\%; $95 \%$ CI 26.2-49.4) studies (Table 4). Detailed subgroup analyses for side, gender, and geographical origin of the study are presented in Table 5. No significant differences were observed in the leave-one-out sensitivity analysis.

\section{Distance of extralaryngeal branching site to the inferior rim of the cricothyroid joint}

Six studies $(n=456$ nerves with ELB) reported extractable data for the distance from the ELB site to the inferior rim of 


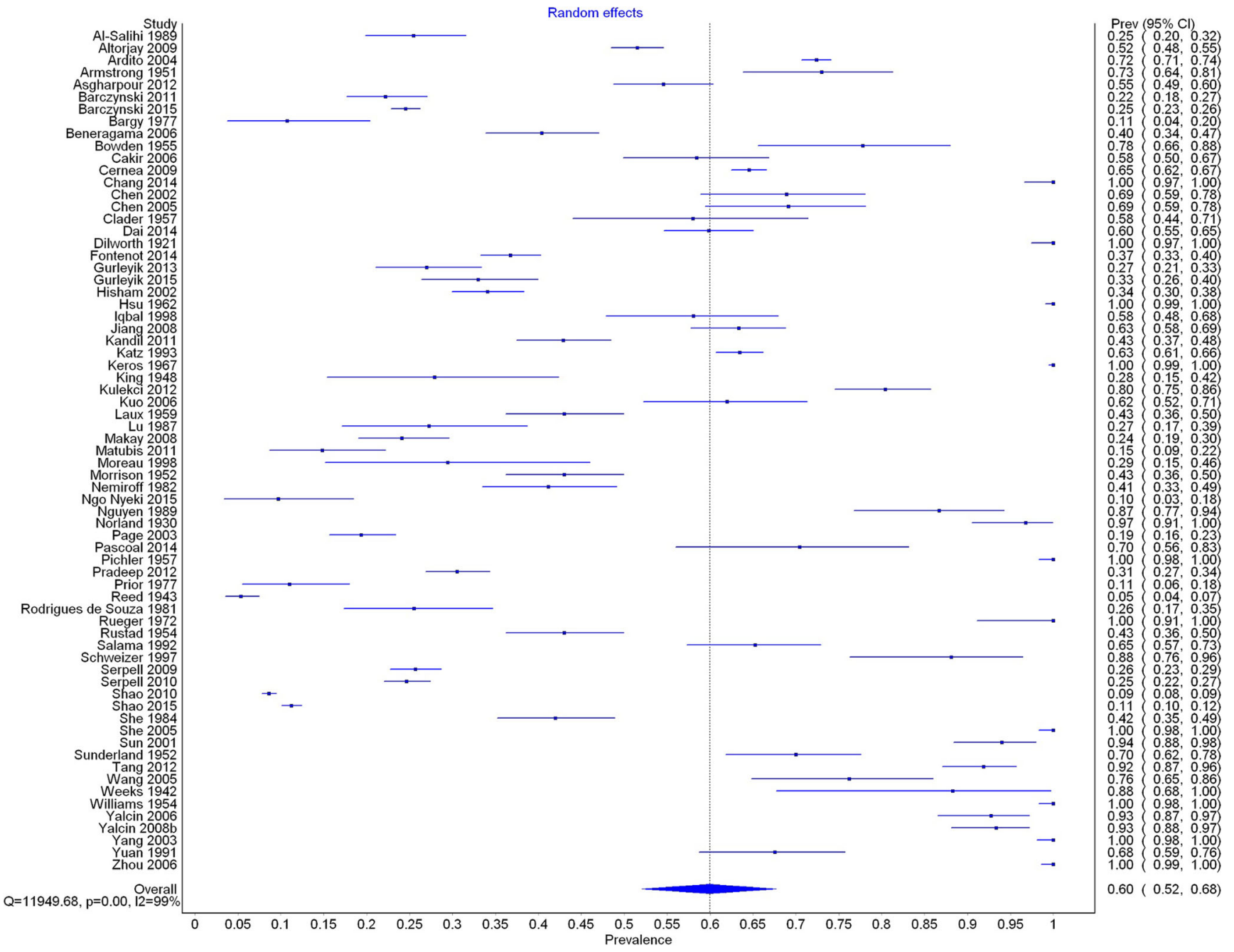

Fig. 2 Forest plot for prevalence of extralaryngeal branching of the recurrent laryngeal nerve

Table 3 Subgroup analysis for the prevalence of extralaryngeal branching

\begin{tabular}{llll}
\hline Subgroup & No. of studies (no. of nerves) & $\begin{array}{l}\text { Pooled prevalence } \\
\text { of ELB \% (95\% CI) }\end{array}$ & $I^{2}$ : \% (95\% CI)* \\
\hline Overall & $69(28,387)$ & $60.0(52.0-67.7)$ & $99.4(99.4-99.5)$ \\
Cadaveric & $42(5250)$ & $73.3(61.0-84.0)$ & $98.9(98.8-99.0)$ \\
Intraoperative & $26(23,071)$ & $39.2(29.0-49.9)$ & $99.6(99.6-99.6)$ \\
Intraoperative (prospective) & $16(14,965)$ & $33.4(20.5-47.7)$ & $99.7(99.6-99.7)$ \\
Intraoperative (retrospective) & $10(8106)$ & $50.2(32.0-68.4)$ & $99.6(99.5-99.6)$ \\
Left sides & $29(6443)$ & $56.6(43.6-69.2)$ & $98.9(98.8-99.1)$ \\
Right sides & $30(6561)$ & $58.5(45.1-71.3)$ & $99.0(98.9-99.1)$ \\
Males & $6(420)$ & $59.6(20.0-87.8)$ & $97.3(95.8-98.2)$ \\
Females & $6(794)$ & $59.7(22.7-92.0)$ & $98.1(97.1-98.7)$ \\
Asia & $23(10,754)$ & $66.1(50.2-80.4)$ & $99.5(99.5-99.6)$ \\
Europe & $24(9417)$ & $62.7(49.2-75.3)$ & $99.3(99.2-99.4)$ \\
North America & $12(3456)$ & $55.8(39.3-71.7)$ & $98.7(98.4-99.0)$ \\
Oceania & $5(2302)$ & $44.3(29.0-60.2)$ & $97.9(96.8-98.7)$ \\
South America & $3(2296)$ & $53.4(25.3-80.5)$ & $96.7(93.3-98.4)$ \\
\hline
\end{tabular}

* $p$ value for Cochran's $\mathrm{Q}$ for all subgroups was $<0.001$ 
Fig. 3 Types of extralaryngeal branching patterns of the recurrent laryngeal nerve with their pooled cadaver prevalence rates. Presented as pooled prevalence rate (95\% confidence interval)
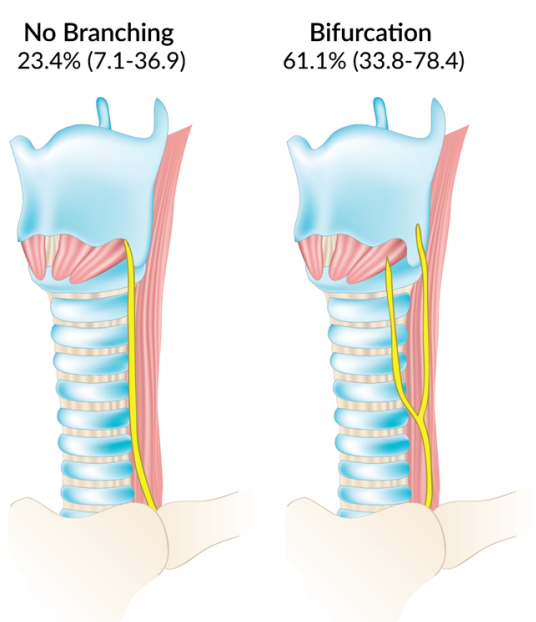

Multiple Branches $6.5 \%(0-15.5)$ the cricothyroid joint. When ELB was present, it occurred most commonly at distance of $1-2 \mathrm{~cm}$, with a pooled prevalence of $74.8 \%$ of cases ( $95 \%$ CI 44.7-94.1), followed by a distance of $0-1 \mathrm{~cm}$ in $15.4 \%$ (95\% CI 0-37.3). Further distance data are presented in Table 6 .

\section{Prevalence of positive motor signals in the extralaryngeal anterior and posterior branches}

A total of five studies [10, 30, 39, 41, 63] $(n=1112$ bifurcated nerves) reported the presence of a positive motor signal in the anterior and posterior branches of an extralaryngeally bifurcated RLN, as assessed electrophysiologically during surgical procedures. A positive motor signal was obtained in $99.9 \%$ (95\% CI 99.7-100.0) of anterior RLN branches $\left(I^{2}=0 \%\right.$ (95\% CI $0-15.4) ; p=0.912)$ but in only $1.5 \%(95 \% \mathrm{CI}$ $0.1-3.9)$ of posterior branches $\left(I^{2}=76.6 \%(95 \%\right.$ CI 43.0 $90.4) ; p=0.002)$. Details of the studies reporting on motor signals are presented in Table 7. To mitigate for any potential differences due to recent technical development and changes in electrophysiological equipment, a subgroup analysis restrictive to studies conducted only within the past 2 years was performed. Three studies [30, 39, 41] $(n=938$ bifurcated nerves) were included in the subgroup analysis. For the anterior branch, a positive motor signal was detected in $99.9 \%$ (95\% CI 99.7-100.0; $I^{2}=0.0 \%$ (95\% CI $\left.0.0-54.3\right)$; $p=0.454$ ) of cases, equivalent to the overall analysis. For the posterior branch, a positive motor signal was detected in $2.6 \%$ (95\% CI $0.2-6.9 ; I^{2}=84.6 \%$ (95\% CI 54.4-94.8); $p=0.001$ ) of cases, slightly greater than the overall analysis, albeit not significantly.

\section{Discussion}

There is wide variability in the ELB of the RLN, and its characteristics have not been assessed completely. The aim of our study was to provide a comprehensive meta-analysis on the ELB variants of the RLN to allow for pertinent clinical applications of the data.

Our results showed that the overall prevalence of ELB was $60.0 \%$. Studies such as Dai et al. [38] and Cakir et al. [33] demonstrated similar findings, whereas other studies have reported prevalences ranging anywhere from $5 \%$ [11] to $100 \%$ [12-21]. Extensive subgroup analysis on the presence of ELB was performed. There were significant differences in the prevalence of ELB between cadaveric studies (73.3\%) and intraoperative studies $(39.2 \%)$. This suggests that the prevalence of ELB could be grossly underestimated in the operating theater. We believe this could be due to difficulty in viewing the branches of the RLN because of localized inflammation, edema, and the small caliber of nerves exhibiting ELB.

Table 4 Type of branching by type of study

\begin{tabular}{lllllll}
\hline & $\begin{array}{l}\text { No. of studies } \\
\text { (no. of nerves) }\end{array}$ & No branching $\%(95 \% \mathrm{CI})$ & $\begin{array}{l}\text { Bifurcation } \% \\
(95 \% \mathrm{CI})\end{array}$ & $\begin{array}{l}\text { Trifurcation \% } \\
(95 \% \mathrm{CI})\end{array}$ & $\begin{array}{l}\text { Multiple branches \% } \\
(95 \% \mathrm{CI})\end{array}$ & $\begin{array}{l}I^{2}: \% \\
(95 \% \mathrm{CI})\end{array}$ \\
\hline Overall & $47(16,618)$ & $42.0(28.1-47.1)$ & $51.1(35.7-55.3)$ & $4.7(1.0-9.2)$ & $2.2(0-5.9)$ & $99.3(99.2-99.4)$ \\
Cadaveric & $27(3361)$ & $23.4(7.1-36.9)$ & $61.1(33.8-78.4)$ & $9.0(0.3-20.9)$ & $6.5(0-15.5)$ & $99.1(99.0-99.2)$ \\
Intraoperative & $19(13,191)$ & $61.3(49.1-72.4)$ & $37.6(26.2-49.4)$ & $1.0(0-4.1)$ & $0.1(0-1.7)$ & $99.4(99.3-99.5)$ \\
Intraoperative (prospective) & $12(6644)$ & $65.4(52.0-77.5)$ & $33.2(21.2-46.4)$ & $1.2(0-5.0)$ & $0.1(0-2.0)$ & $99.0(98.8-99.2)$ \\
Intraoperative (retrospective) & $8(6613)$ & $56.8(35.5-75.9)$ & $42.1(22.3-62.4)$ & $0.7(0-6.2)$ & $0.4(0-5.1)$ & $99.5(99.4-99.6)$ \\
\hline
\end{tabular}

* $p$ value for Cochran's $Q$ for all subgroups was $<0.001$ 
Table 5 Type of branching by side, gender, and geographical origin

\begin{tabular}{lllllll}
\hline & $\begin{array}{l}\text { No. of studies } \\
\text { (no. of nerves) }\end{array}$ & $\begin{array}{l}\text { No branching } \% \\
(95 \% \mathrm{CI})\end{array}$ & $\begin{array}{l}\text { Bifurcation } \% \\
(95 \% \mathrm{CI})\end{array}$ & $\begin{array}{l}\text { Trifurcation \% } \\
(95 \% \mathrm{CI})\end{array}$ & $\begin{array}{l}\text { Multiple branches \% } \\
(95 \% \mathrm{CI})\end{array}$ & $I^{2}: \%(95 \%$ CI)* \\
\hline Overall & $47(16,618)$ & $42.0(28.1-47.1)$ & $51.1(35.7-55.3)$ & $4.7(1.0-9.2)$ & $2.2(0-5.9)$ & $99.3(99.2-99.4)$ \\
Left sides & $26(3942)$ & $50.7(33.2-60.6)$ & $38.9(23.2-49.6)$ & $6.3(0.7-14.2)$ & $4.1(0-9.8)$ & $98.5(98.2-98.7)$ \\
Right sides & $26(4262)$ & $45.5(28.0-56.3)$ & $43.4(26.2-54.3)$ & $6.8(0.8-15.4)$ & $4.3(0-10.5)$ & $98.7(98.5-98.9)$ \\
Males & $5(362)$ & $39.8(4.5-70.7)$ & $46.8(8.0-76.2)$ & $9.0(0-31.8)$ & $4.3(0-23.0)$ & $97.5(96.1-98.5)$ \\
Females & $5(754)$ & $39.6(0-76.6)$ & $49.1(3.6-85.6)$ & $6.2(0-33.2)$ & $5.1(0-30.8)$ & $98.4(97.6-98.9)$ \\
Asia & $19(5849)$ & $35.2(13.1-49.4)$ & $49.9(23.1-62.2)$ & $8.1(0-18.6)$ & $6.8(0-16.6)$ & $99.4(99.4-99.5)$ \\
Europe & $16(5624)$ & $35.7(18.6-48.4)$ & $59.0(38.0-69.6)$ & $3.8(0-10.5)$ & $1.5(0-6.3)$ & $99.1(98.9-99.2)$ \\
North America & $7(2007)$ & $53.0(31.3-71.8)$ & $43.4(22.8-62.9)$ & $2.6(0-10.9)$ & $1.0(0-7.1)$ & $98.6(98.1-99.0)$ \\
Oceania & $3(1195)$ & $57.7(33.5-79.2)$ & $37.0(15.7-60.3)$ & $5.3(0-17.9)$ & $0.1(0-4.4)$ & $97.7(95.7-98.8)$ \\
\hline
\end{tabular}

* $p$ value for Cochran's $Q$ for all subgroups was $<0.001$

Furthermore, the inability to completely dissect the small branches during operations and surgeons not addressing the small "accessory" RLN branches as ELB may also contribute to the large gap between cadaveric vs. intraoperative prevalence. Some intraoperative studies noted that only nerves which bifurcated and entered at the lower margin of the larynx were indeed counted as branched nerves (ELB) [30,39]. An intraoperative study by Gurleyik in 2013 [40] noted that many of the small branches of the RLN that may be found in cadaveric studies are simply not perceivable during surgical procedures. As is mentioned in a study by Ngo Nyeki in 2015 [54], intraoperative assessment of ELB was not systematically investigated. Regardless, diligent assessment and dissection are needed during surgical procedures to avoid iatrogenic injuries and complications. Future research regarding RLN ELB needs to be meticulous, particularly with regard to intraoperative studies. We believe that, to date, the true prevalence has been better reflected in the results of cadaveric-based studies. Furthermore, detailed morphometric analysis could be performed on the RLN to determine which, if any, caliber nerve is likely to have ELB.

No major deviations from the overall prevalence were noted in geographic, sex-based, and laterality subgroup analyses, and thus, all patients should be considered to have equal risk of ELB. We posit that this is a logical finding, given that the

Table 6 Distance from the extralaryngeal branching site to the inferior rim of the cricothyroid joint

\begin{tabular}{ll}
\hline Distance $(\mathrm{cm})$ & Pooled prevalence \% $(95 \% \mathrm{CI})$ \\
\hline $0-1$ & $15.4(0-37.3)$ \\
$1-2$ & $74.8(44.7-94.1)$ \\
$2-3$ & $6.0(0-22.2)$ \\
$3-4$ & $3.8(0-17.7)$ \\
\hline
\end{tabular}

Six studies (456 nerves with ELB), $I^{2}=97.1 \%$ (95\% CI 95.4-98.1), $p<0.001$ embryological development of these structures is largely uniform, barring any unique developmental pathologies. Importantly, surgeons need to refrain from assuming that the presence of ELB is always purely symmetrical. We note that a mere $36.5 \%$ of RLNs had symmetrical branching.

A subset of the studies [4-7, 9-21, 26-30, 32-36, 38-43, $47,48,50-52,54,55,59,60,62,63,66,68,71,73,75-82]$ reporting information on ELB also reported information on the type of branching. In those studies, bifurcation was the most common pattern observed, with a pooled prevalence of $51.1 \%$ of nerves. The second most common pattern was the lack of branching, followed by trifurcation and multiple branches. We note that due to the potential lack of systematic investigation of ELB during intraoperative procedures, there may be underreporting of non-bifurcating patterns (i.e., trifurcation, multiple branches) and ELB in general. As was mentioned previously, some surgeons only considered ELB to be present in cases of bifurcation as is seen in Barczyński et al. [30] and Fontenot et al. [39], and thus, some patterns of trifurcation and multiple branching may have been overlooked or not have been noticeable.

A small number of studies reported on the distance of the branching point of the RLN from the inferior rim of the cricothyroid joint (CTJ). Most of the nerves $(90.2 \%)$ branched within the proximal $2 \mathrm{~cm}$ of the CTJ, suggesting that some intraoperative studies have failed to note incidences of branching if it occurred in such close proximity to the larynx. With the overwhelming number of nerves branching at this distance from the cricothyroid joint, it is evident how essential it is to successfully identify the RLN in the surgical field.

Motor signaling was assessed in both anterior and posterior divisions of the RLN. It was noted that nearly $100 \%$ of anterior branches had positive motor signaling, but it was found posteriorly in only $1.5 \%$. This supports the notion that the anterior branch is almost the sole supplier of motor innervation to the RLN-innervated muscles of the larynx, the sensory fibers traveling in the posterior division [10]. However, our 
Table 7 Motor signaling in extralaryngeal branches of the recurrent laryngeal nerve

\begin{tabular}{|c|c|c|c|c|}
\hline Study ID & Method of signal detection & $\begin{array}{l}n \text { (number of RLN } \\
\text { with ELB) }\end{array}$ & $\begin{array}{l}\text { Positive motor signal } \\
\text { in anterior branch }(\%)\end{array}$ & $\begin{array}{l}\text { Positive motor signal } \\
\text { in posterior branch }(\%)\end{array}$ \\
\hline Barczyński et al. [30] & $\begin{array}{l}\text { NIM } 2.0 \text { followed by the NIM } 3.0 \text { system (Medtronic } \\
\text { USA, Inc., Jacksonville, FL) at } 1 \mathrm{~mA}\end{array}$ & 613 & $613(100 \%)$ & $8(1.3 \%)$ \\
\hline Gurleyik [41] & $\begin{array}{l}\text { IONM device. Nerve Integrity Monitor (NIM-Response } \\
\text { 3.0 System; Medtronic Xomed, Jacksonville, FL) at } \\
1 \mathrm{~mA}\end{array}$ & 61 & $61(100 \%)$ & $7(11.5 \%)$ \\
\hline Fontenot et al. [39] & $\begin{array}{l}\text { IONM device (Xomed NIM System; Medtronic USA, } \\
\text { Inc., Jacksonville, FL) at } 1.0 \mathrm{~mA}\end{array}$ & 264 & $264(100 \%)$ & $3(1.1 \%)$ \\
\hline Kandil et al. [10] & $\begin{array}{l}\text { IONM device (Xomed NIM System; Medtronic USA, } \\
\text { Inc., Jacksonville, FL) at } 0.5 \mathrm{~mA}\end{array}$ & 133 & $133(100 \%)$ & $0(0 \%)$ \\
\hline Serpell et al. [63] & $\begin{array}{l}\text { IONM device (Xomed NIM System; Medtronic USA, } \\
\text { Inc., Jacksonville, FL) }\end{array}$ & 41 & $41(100 \%)$ & $0(0 \%)$ \\
\hline
\end{tabular}

analysis notes that up to $3.9 \%$ of posterior branches could contain some motor fibers and, as such, care should be taken to protect them whenever possible. Additional problems arise when looking at the rapid development and honing of the technology and devices used to identify these signals. The more recent studies included in our analysis [30, 41] tended to have higher incidence of motor signaling in the posterior branches leading to the conclusion that signals in this division may have been previously underreported due to devices simply not being sensitive enough.

Injury to the RLN is one of the most feared and challenging postoperative complications in thyroid surgery, with $6 \%$ experiencing temporary deficits and $1 \%$ experiencing permanent nerve palsy $[76,77]$. With the realization that over half of patients have some element of ELB, precautions should be taken to prevent these iatrogenic complications directly. We recommend that surgeons attempt to expose the RLN completely, along with any of its early bifurcating terminal branches if they are present [41]. However, not all surgeons recommend complete dissection of the RLN, as it may require a more invasive procedure [54].

The use of anatomical landmarks such as the nerve's relationship with the inferior thyroid artery, ligament of Berry, tracheoesophageal groove, or tubercle of Zuckerkandl may be helpful for determining the RLN's location but are often highly variable in their anatomical relationship to the nerve [40, 54, 78]. These relationships may be further complicated by present pathology such as a large goiter or inflammation and edema altering the normal anatomy, making nerve identification more difficult. The use of intraoperative nerve monitoring (IONM) devices has shown potential; however, to date, it is not significantly more effective at reducing iatrogenic injuries as compared to nerve visualization [79, 80]. We make the proposition that IONM be used in instances where patients may have an underlying pathology such as large goiter or inflammation which restricts visualization of the necessary structures or, when patients are undergoing reoperation, as scar tissue can make nerve identification difficult [81]. As is noted in the German Association of Endocrine Surgeons' guidelines for thyroid disease, the use of IONM should serve as a complimentary tool for surgeons for ensuring the identification and protection of the RLN during operative procedures [83]. Further described by Musholt et al. [83] is that IONM is indispensable in the prevention of bilateral RLN injuries which result in severe deficits. Another option available to surgeons is the use of pre-operative ultrasonography (USG) to identify structures and potential anatomical variants. Rare variants such as nonrecurrent laryngeal nerves have been successfully identified using USG $98 \%$ of the time, and thus, this method may be helpful in reducing the risk of iatrogenic injury to the RLN [82]. Konschake et al. [84] and Gong et al. [85] note the use of USG as the most efficient and effective way of preoperatively identifying these variants and avoiding unnecessary radiation exposure. The use of pre-operative USG to identify ELB should be evaluated in future studies.

Our meta-analysis on the ELB of the RLN was limited by a number of factors, such as unclear or difficult-to-interpret data and the lack of detailed information on nerve branching patterns, which resulted in the exclusion of several studies. Additionally, there was high heterogeneity among studies, which persisted despite extensive subgroup analysis, suggesting it could be attributed to the intrinsic variability of the RLN. Further limitation factors included the lack of a quality assessment and risk of bias tool for anatomical studies and a lack of assessment of publication bias because there was no statistical measure for prevalence meta-analysis. Throughout the study, authors were contacted when necessary and possible in an attempt to resolve discrepancies, provide clarification, and minimize bias.

In conclusion, the RLN is highly variable and has a high prevalence of ELB. The RLN in most of the population has ELB in the form of bifurcation, followed in prevalence by no branching, trifurcation, and multiple branching. Extralaryngeal branching, if present, is typically within $2 \mathrm{~cm}$ of the inferior rim of the cricothyroid joint, with the overwhelming majority of anterior branches containing the motor 
fibers and posterior branches the sensory fibers. The high prevalence of ELB needs to be factored into the assessment and operative procedure of every patient. Only a proper and complete understanding of the variant anatomy of the RLN can provide for the best chance of a complication- and injuryfree procedure.

Acknowledgments Krzysztof A. Tomaszewski was supported by the Foundation for Polish Science (FNP). We wish to thank Karolina Saganiak for the anatomical drawings used in this manuscript.

\section{Compliance with ethical standards}

Funding The study was supported by the statutory funds of Jagiellonian University Medical College.

Conflict of interest The authors declare that they have no competing interests.

Open Access This article is distributed under the terms of the Creative Commons Attribution 4.0 International License (http:// creativecommons.org/licenses/by/4.0/), which permits unrestricted use, distribution, and reproduction in any medium, provided you give appropriate credit to the original author(s) and the source, provide a link to the Creative Commons license, and indicate if changes were made.

\section{References}

1. Moore KL, Dalley AF, Agur AMR (2014) Clinically oriented anatomy, 7th edn. Lippincott Williams \& Wilkins, Philadelphia

2. Miyauchi A, Masuoka H, Nakayama A, Higashiyama T (2015) Innervation of the cricothyroid muscle by extralaryngeal branches of the recurrent laryngeal nerve. Laryngoscope

3. Asgharpour E, Maranillo E, Sañudo J et al (2012) Recurrent laryngeal nerve landmarks revisited. Head Neck 34:1240-1246

4. Beneragama T, Serpell JW (2006) Extralaryngeal bifurcation of the recurrent laryngeal nerve: a common variation. ANZ J Surg 76: 928-931

5. Nemiroff PM, Katz AD (1982) Extralaryngeal divisions of the recurrent laryngeal nerve. Surgical and clinical significance. Am J Surg 144:466-469

6. Salama AB, McGrath P (1992) Recurrent laryngeal nerve and the posterior fascial attachment of the thyroid gland. Aust N Z J Surg 62:444-449

7. Schweizer V, Dörfl J (1997) The anatomy of the inferior laryngeal nerve. Clin Otolaryngol Allied Sci 22:362-369

8. He X, Sun J, Ye C et al (2000) Anatomic study on the nervous distribution of the human. J Clin Otorhinolaryngol Head Neck Surg 14:387-389

9. Makay O, Icoz G, Yilmaz M et al (2008) The recurrent laryngeal nerve and the inferior thyroid artery - anatomical variations during surgery. Langenbecks Arch Surg 393:681-685

10. Kandil E, Abdelghani S, Friedlander P et al (2011) Motor and sensory branching of the recurrent laryngeal nerve in thyroid surgery. Surgery 150:1222-1227

11. Reed AF (1943) The relations of the inferior laryngeal nerve to the inferior thyroid artery. Anat Rec 85:17-23

12. Chang S (2014) The analysis of the significance of the recurrent laryngeal nerve in the applied anatomy of neck surgery. China Pract Med 9:72-73
13. Dilworth TF (1921) The nerves of the human larynx. J Anat 56:48 52

14. Hsu YH, Liu YH, Leng TC (1962) Anatomical study of the recurrent laryngeal nerve. Chin Med J (Engl) 81:481-484

15. Keros P, Nemanić D (1967) The terminal branching of the recurrent laryngeal nerve. Pract Otorhinolaryngol 29:5-10

16. Pichler H, Gisel A (1957) The clinical significance of the ramification of the recurrent laryngeal nerves; a critical anatomical study. Laryngoscope 67:105-117

17. Rueger RS (1972) The superior laryngeal nerve and the interarytenoid muscle in humans: an anatomical study. Laryngoscope 82:2008-2031

18. She Y, Zhang Z, Wu J et al (2005) Applied anatomy of the superior and recurrent laryngeal nerves. J North Sichuan Med Coll 20:1-3

19. Williams AF (1954) The recurrent laryngeal nerve and the thyroid gland. J Laryngol Otol 68:719-725

20. Yang C, Zhou L, Zeng Z et al (2003) Clinical practice of the recurrent laryngeal nerve in the neck dissection. Hei Long Jiang Med J 27:508

21. Zhou X, Huang H, Li Z et al (2006) An applied anatomical study of recurrent laryngeal nerve. Sichuan J Anat 14:22-24

22. Moher D, Liberati A, Tetzlaff J, Altman DG (2009) Preferred reporting items for systematic reviews and meta-analyses: the PRISMA statement. Ann Intern Med 151(264-9):W64

23. Henry BM, Tomaszewski KA, Walocha JA (2016) Methods of evidence-based anatomy: a guide to conducting systematic reviews and meta-analysis of anatomical studies. Ann Anat. doi:10.1016/j. aanat.2015.12.002

24. Higgins J, Green S (2011) Cochrane handbook for systematic reviews of interventions. The Cochrane Collaboration

25. Al-Salihi AR, Dabbagh AW (1989) Anatomy of the recurrent laryngeal nerve in normal Iraqis. Acta Anat (Basel) 135:245-247

26. Altorjay A, Tihanyi Z, Luka F et al (2009) Place and value of the recurrent laryngeal nerve (RLN) palpatory method in preventing RLN palsy during thyroid surgery. Head Neck 31:538-547

27. Ardito G, Revelli L, D'Alatri L et al (2004) Revisited anatomy of the recurrent laryngeal nerves. Am J Surg 187:249-253

28. Armstrong WG, Hinton JW (1951) Multiple divisions of the recurrent laryngeal nerve. An anatomic study. AMA Arch Surg 62:532539

29. Barczyński M, Konturek A, Stopa M et al (2011) Clinical value of intraoperative neuromonitoring of the recurrent laryngeal nerves in improving outcomes of surgery for well-differentiated thyroid cancer. Pol Przegl Chir 83:196-203

30. Barczyński M, Stopa M, Konturek A, Nowak W (2015) The overwhelming majority but not all motor fibers of the bifid recurrent laryngeal nerve are located in the anterior extralaryngeal branch. World J. Surg

31. Bargy F, Calmat A, Leguerrier A et al (1977) Study of the vascular density of tissues surrounding the right recurrent nerve (apropos of 56 cases). Bull Assoc Anat (Nancy) 61:311-316

32. Bowden RE (1955) The surgical anatomy of the recurrent laryngeal nerve. Br J Surg 43:153-163

33. Cakir BO, Ercan I, Sam B, Turgut S (2006) Reliable surgical landmarks for the identification of the recurrent laryngeal nerve. Otolaryngol Head Neck Surg 135:299-302

34. Cernea CR, Hojaij FC, De Carlucci D et al (2009) Recurrent laryngeal nerve: a plexus rather than a nerve? Arch Otolaryngol Head Neck Surg 135:1098-1102

35. Chen X, Xie L, Guo X (2002) The regional dissection and clinical application in pars cervicalis of recurrent laryngeal nerve. Sichuan J Anat 10:150-152

36. Chen T, Hu Q, Ou X et al (2005) Applied anatomy of the recurrent laryngeal nerve and inferior thyroid artery. Anat Res 27:139-140 
37. Clader DN, Luter PW, Daniels BT (1957) A photographic study of the superior and inferior laryngeal nerves and the superior and inferior thyroid arteries. Am Surg 23:609-618

38. Dai H, Hua Q, Jiang Y, Sheng J (2014) Anatomy of recurrent laryngeal nerve during thyroid surgery. Lin Chung Er Bi Yan Hou Tou Jing Wai Ke Za Zhi 28:1925-1926, 1930

39. Fontenot TE, Randolph GW, Friedlander PL et al (2014) Gender, race, and electrophysiologic characteristics of the branched recurrent laryngeal nerve. Laryngoscope 124:2433-2437

40. Gurleyik E (2013) Extralaryngeal terminal division of the inferior laryngeal nerve: anatomical classification by a surgical point of view. J Thyroid Res 2013:731250

41. Gurleyik E (2015) Location of motor fibers within branches of the recurrent laryngeal nerve with extralaryngeal terminal bifurcation; Functional identification by intraoperative neuromonitoring. Surgery

42. Hisham AN, Lukman MR (2002) Recurrent laryngeal nerve in thyroid surgery: a critical appraisal. ANZ J Surg 72:887-889

43. Iqbal J, Zubair M (1998) Anatomical considerations regarding recurrent laryngeal nerve in relation with thyroid surgery. J Coll Physicians Surg Pak 8:276-277

44. Jiang Y, Dong P, Li X et al (2008) The significance of anastomosing the recurrent laryngeal nerve in the thyroidectomy. Lin Chung Er Bi Yan Hou Tou Jing Wai Ke Za Zhi 22:544-546, 550

45. Katz AD, Nemiroff P (1993) Anastamoses and bifurcations of the recurrent laryngeal nerve - report of 1177 nerves visualized. Am Surg 59:188-191

46. King BT, Gregg RL (1948) An anatomical reason for the various behaviors of paralyzed vocal cords. Ann Otol Rhinol Laryngol 57: 925-944

47. Kulekci M, Batioglu-Karaaltin A, Saatci O, Uzun I (2012) Relationship between the branches of the recurrent laryngeal nerve and the inferior thyroid artery. Ann Otol Rhinol Laryngol 121:650 656

48. Kuo X, Yi D, Zhang W (2006) Applied anatomy of the recurrent laryngeal nerve. Anat Res 28:222-224

49. Laux G, Guerrier Y (1959) L'innervation des muscles du larynx. C R Assoc Anat 46:46-62

50. Lu X, Ouyang Z, Chou X et al (1987) Relationship of inferior cornu of the thyroid cartilage with the point of entry of recurrent laryngeal nerve into the larynx and its clinical significance. Chin J Gen Surg $25: 212-214$

51. Matubis JS, Dumlao KJP, Carrillo RJC (2011) The recurrent laryngeal nerve in relation to the inferior thyroid artery in adult Filipino cadavers. Philipp J Otolaryngol Head Neck Surg 1:13-17

52. Moreau S, Goullet de Rugy M, Babin E et al (1998) The recurrent laryngeal nerve: related vascular anatomy. Laryngoscope 108: $1351-1353$

53. Morrison LF (1952) Recurrent laryngeal nerve paralysis - revised conception based on a dissection of 100 cadavers. Ann Otol Rhinol Laryngol 61:567-592

54. Ngo Nyeki A-R, Njock L-R, Miloundja J et al (2015) Recurrent laryngeal nerve landmarks during thyroidectomy. Eur. Ann. Otorhinolaryngol. Head Neck Dis

55. Nguyen M, Junien-Lavillauroy C, Faure C (1989) Anatomical intra-laryngeal anterior branch study of the recurrent (inferior) laryngeal nerve. Surg Radiol Anat 11:123-127

56. Nordland M (1930) The larynx as related to surgery of the thyroid. Based on an anatomical study. Surg Gynecol Obstet 51:449-459

57. Page C, Foulon P, Strunski V (2003) The inferior laryngeal nerve: surgical and anatomic considerations. Report of 251 thyroidectomies. Surg Radiol Anat 25:188-191

58. Pascoal AAF, Fernandes JR, Ruiz CR et al (2014) Terminal branch of recurrent human laryngeal nerve. Adv Anat
59. Pradeep PV, Jayashree B, Harshita SS (2012) A closer look at laryngeal nerves during thyroid surgery: a descriptive study of 584 nerves. Anat Res Int 2012:490390

60. Prior C, Fasce M (1977) The relation between the recurrent nerve and the inferior thyroid artery. Arch Maragliano Patol Clin 33:29 36

61. de Souza RR, de Carvalho CA, Chih CL, de Andrade AC (1981) Early division of the recurrent laryngeal nerve and its branch communicating with the superior laryngeal nerve in man; ethnic and anatomo-surgical study. Rev Hosp Clin Fac Med Sao Paulo 36: $258-260$

62. Rustad WH (1954) Revised anatomy of the recurrent laryngeal nerves: surgical importance, based on the dissection of $100 \mathrm{ca}-$ davers. J Clin Endocrinol Metab 14:87-96

63. Serpell JW, Yeung MJ, Grodski S (2009) The motor fibers of the recurrent laryngeal nerve are located in the anterior extralaryngeal branch. Ann Surg 249:648-652

64. Serpell JW (2010) New operative surgical concept of two fascial layers enveloping the recurrent laryngeal nerve. Ann Surg Oncol 17:1628-1636

65. Shao T, Yang W, Zhang T et al (2010) A newly identified variation at the entry of the recurrent laryngeal nerve into the larynx. J Invest Surg 23:314-320

66. Shao T, Qiu W, Yang W (2015) Confirmation of an anatomic variation of the recurrent laryngeal nerve at site of entry into the larynx in Chinese population. Am J Otolaryngol

67. She B, Sheng P, Zhang G (1984) Anatomic study of the recurrent laryngeal nerve. Acad J Guangzhou Med Coll 12:31-37

68. Sun SQ, Zhao J, Lu H et al (2001) An anatomical study of the recurrent laryngeal nerve: its branching patterns and relationship to the inferior thyroid artery. Surg Radiol Anat 23:363-369

69. Sunderland S, Swaney WE (1952) The intraneural topography of the recurrent laryngeal nerve in man. Anat Rec 114:411-426

70. Tang W-J, Sun S-Q, Wang X-L et al (2012) An applied anatomical study on the recurrent laryngeal nerve and inferior thyroid artery. Surg Radiol Anat 34:325-332

71. Wang L, Zhu L, Wang M, Liu F (2005) Anatomic study on the recurrent laryngeal nerve in thyroid surgery. Lin Chuang Er Bi Yan Hou Ke Za Zhi 19:112-113

72. Weeks C, Hinton JW (1942) Extralaryngeal division of the recurrent laryngeal nerve: its significance in vocal cord paralysis. Ann Surg 116:251-258

73. Yalçin B, Tuğcu H, Cantürk N, Ozan H (2006) Laryngeal branching pattern of the inferior laryngeal nerve, before entering the larynx. Surg Radiol Anat 28:339-342

74. Yalcin B, Tunali S, Ozan H (2008) Extralaryngeal division of the recurrent laryngeal nerve: a new description for the inferior laryngeal nerve. Surg Radiol Anat 30:215-220

75. Yuan S (1991) Anatomy and clinical significance of the recurrent laryngeal nerve. J Clin Otorhinolaryngol Head Neck Surg 5:4-7

76. Lo CY, Kwok KF, Yuen PW (2000) A prospective evaluation of recurrent laryngeal nerve paralysis during thyroidectomy. Arch Surg 135:204-207

77. Hundahl SA, Cady B, Cunningham MP et al (2000) Initial results from a prospective cohort study of 5583 cases of thyroid carcinoma treated in the united states during 1996. U.S. and German Thyroid Cancer Study Group. An American College of Surgeons Commission on Cancer Patient Care Evaluation study. Cancer 89: 202-217

78. Rajapaksha A, Fernando R, Ranasinghe N, Iddagoda S (2015) Morphology of the tubercle of Zuckerkandl and its importance in thyroid surgery. Ceylon Med J 60:23-24

79. Pisanu A, Porceddu G, Podda M et al (2014) Systematic review with meta-analysis of studies comparing intraoperative neuromonitoring of recurrent laryngeal nerves versus visualization alone during thyroidectomy. J Surg Res 188:152-161 
80. Gremillion G, Fatakia A, Dornelles A, Amedee RG (2012) Intraoperative recurrent laryngeal nerve monitoring in thyroid surgery: is it worth the cost? Ochsner J 12:363-366

81. Barczyński M, Konturek A, Pragacz K et al (2014) Intraoperative nerve monitoring can reduce prevalence of recurrent laryngeal nerve injury in thyroid reoperations: results of a retrospective cohort study. World J Surg 38:599-606

82. Iacobone M, Citton M, Pagura G et al (2015) Increased and safer detection of nonrecurrent inferior laryngeal nerve after preoperative ultrasonography. Laryngoscope 125:1743-1747
83. Musholt TJ, Clerici T, Dralle H et al (2011) German Association of Endocrine Surgeons practice guidelines for the surgical treatment of benign thyroid disease. Langenbeck's Arch Surg / Dtsch Gesellschaft für Chir 396:639-649

84. Konschake M, Zwierzina ME, Pechriggl EJ et al (2016) The nonrecurrent laryngeal nerve: a clinical anatomic mapping with regard to intraoperative neuromonitoring. Surgery

85. Gong R, Luo S, Gong Y et al (2014) Prediction of nonrecurrent laryngeal nerve before thyroid surgery-experience with 1825 cases. J Surg Res 189:75-80 